\title{
Duplicated Internal Auditory Canal: High-Resolution CT and MRI Findings
}

\author{
Linsheng Wang, $M D^{1}$, Lihong Zhang, $M D^{2}$, Xian Li, $M D^{1}$, Xiang Guo, MD \\ ${ }^{1}$ Imaging Center, the Affiliated Hospital of Jining Medical University, Jining, China; ${ }^{2}$ Department of Radiology, the First People's Hospital of \\ Jining City, Jining, China
}

Objective: To summarize the high-resolution computed tomography (HRCT) and magnetic resonance imaging (HRMRI) features of duplicated internal auditory canals (DIACs).

Materials and Methods: Ear HRCT data of 64813 patients with sensorineural hearing loss (SNHL), obtained between August 2009 and November 2017, were reviewed. Among these patients, 12 (13 ears) were found to have DIACs, 9 of whom underwent HRMRI. Their images were evaluated by two otoradiologists.

Results: The rate of occurrence of DIAC among SNHL patients was $0.019 \%(12 / 64813)$. The internal auditory canals of 13 ears were divided into double canals by complete $(n=6)$ and incomplete $(n=7)$ bony septa, with varied orientations ranging from horizontal to approximately vertical. All of the anterosuperior canals extended into the facial nerve (FN) canal, except for 1 , which also extended to the vestibule. The posteroinferior canals ended in the cochlea and vestibule, except for 2, which also connected to the FN canals. Magnetic resonance images revealed that $77.8 \%(7 / 9)$ and $22.2 \%(2 / 9)$ of vestibulocochlear nerves (VCNs) were aplastic and hypoplastic, respectively. Furthermore, $88.9 \%(8 / 9)$ of FNs were normal, except for 1 , which was hypoplastic. All of the affected ears also had other ear anomalies: a narrow, bony cochlear nerve canal was the most common other anomaly, accounting for $92.3 \%$ (12/13). Malformations of other systems were not found.

Conclusion: Double-canal appearance is a characteristic finding of DIAC on HRCT, and it is usually accompanied by other ear anomalies. The VCN usually appears aplastic, with a normal FN, on HRMRI.

Keywords: Internal auditory canal; Duplication; Computed tomography; Magnetic resonance imaging

\section{INTRODUCTION}

A duplicated internal auditory canal (DIAC) is a rare subtype of internal auditory canal (IAC) anomalies and is usually associated with ipsilateral congenital sensorineural hearing loss (SNHL). Thus far, 44 DIAC cases have been reported (1-24), but reports on their imaging features are limited. DIAC is frequently misdiagnosed as IAC stenosis in clinical practice because of lack of knowledge about

Received January 24, 2018; accepted after revision December 2, 2018.

Corresponding author: Lihong Zhang, MD, Department of Radiology, the First People's Hospital of Jining City, No.6 Jiankang Road, Jining 272011, China.

- Tel: (86537) 2902253015 - Fax: (86537) 2253449

- E-mail: zlh968968@163.com

This is an Open Access article distributed under the terms of the Creative Commons Attribution Non-Commercial License (https://creativecommons.org/licenses/by-nc/4.0) which permits unrestricted non-commercial use, distribution, and reproduction in any medium, provided the original work is properly cited. its imaging features $(9,13,21)$. Vestibulocochlear nerves (VCNs) or cochlear nerves (CNs) in stenotic IACs are usually dysplastic; as such, most of the patients can undergo and benefit from electronic cochlear implantation (ECI) $(21,25)$. In comparison, in DIAC, these nerves usually appear aplastic; thus, most of the patients do not achieve marked benefits from $\operatorname{ECI}(20,24)$. Hence, preoperative accurate diagnosis of DIAC through computed tomography (CT) and magnetic resonance imaging (MRI) is necessary to facilitate selection of the appropriate treatment. A preoperative understanding of the accompanying temporal bone anomalies, such as outer, middle, and inner ear malformation and facial nerve (FN) canal migration, is considerably useful for otologists to be able to design appropriate operative approaches for DIAC patients who are suitable for ECI (26).

Therefore, we retrospectively analyzed the imaging data of 12 DIAC patients (13 ears) admitted to our institution over the past 8 years. The purpose of this study was to 
summarize the imaging features of these ears on highresolution CT (HRCT) and HRMRI.

\section{MATERIALS AND METHODS}

Our Institutional Review Board approved this retrospective study and waived the requirement for obtaining informed patient consent.

\section{Study Population}

The ear HRCT images of 64813 SNHL patients diagnosed from August 2009 to November 2017 were retrieved from a picture archiving and communication system in our institution by an experienced otoradiologist. Among these, 12 patients (13 ears) were diagnosed with DIACs according to the following morphological criteria, based on HRCT images: 1) the IAC was divided into 2 or 3 canals by a complete or incomplete bony septum; 2) the duplicated canals were connected to the FN canal or the vestibule and the cochlea. Among these patients, patient 11 had been previously reported (24).

The age of the DIAC patients ranged from 4 months to 80 years ( 4 males and 8 females) (Table 1). Ipsilateral hearing impairment since childhood was the main clinical presentation $(n=10)$; two of these patients also had tinnitus or vertigo. The two other cases were referred to our hospital for microtia and external auditory canal (EAC) atresia, and one of them had left facial palsy since birth. Auditory brainstem responses revealed that all of the affected ears presented a variable degree of SNHL, ranging from moderate to profound (27). The Caroli test indicated mild vestibular weakness in two ears. FN examination showed left peripheral facial palsy (House-Brackmann grade IV) in one affected ear. Other results were normal. None of the patients had a family history of DIAC.

\section{CT and MRI Acquisition}

A 64-section CT scanner (Light Speed 64, GE Healthcare, Milwaukee, WI, USA) was used with the following parameters: $100 \mathrm{kV}$ or $120 \mathrm{kV}$ and $120 \mathrm{mAs}$ or $150 \mathrm{mAs}$; pitch: 0.5371:1; slice thickness: $0.625 \mathrm{~mm}$; reconstruction interval: $0.3 \mathrm{~mm}$; window width and window level: $4000 \mathrm{HU}$ and $650 \mathrm{HU}$, respectively; and field-of-view: $250 \times 250 \mathrm{~mm}$.

A MAGNETOM (1.5T) (Siemens Healthineers, Erlangen, Germany) was utilized for magnetic resonance scanning in 9 patients. The parameters were as follows: three-dimensional (3D) sampling perfection with application-optimized contrasts using different flip angle evolutions sequence, repetition time $=2200 \mathrm{~ms}$, echo time $=264 \mathrm{~ms}$, flip angle $=150^{\circ}$, matrix $=324 \times 320$, field-of-view $=12 \mathrm{~cm}$, slice thickness $=0.5 \mathrm{~mm}$, signal-to-noise ratio $=1.0$, and number of excitations $=2$. On an AW 4.3 (GE Healthcare), the axial multiple planar reformation (MPR) CT image of the bony CN canal (through the mid-modiolar section), the oblique axial MPR (parallel to the long axis of duplicated canals), the oblique coronal MPR (parallel to the long axis of duplicated canals), and the parasagittal MPR (perpendicular to the long axis of duplicated canals), as well as the volume rendering CT images and MRI (threshold: 100-600 HU), were reconstructed.

Table 1. Clinical Presentations of 12 Patients with Duplicated Internal Auditory Canals

\begin{tabular}{|c|c|c|c|c|c|c|}
\hline No. & Sex/Age & Side & Clinical Presentation & $A B R$ & Caroli Test & FNE \\
\hline 1 & $M / 23 y$ & $\mathrm{R}$ & Hearing loss since childhood & Profound & - & - \\
\hline 2 & $F / 80$ y & $\mathrm{R}$ & Hearing loss since childhood & Profound & - & - \\
\hline \multirow{2}{*}{3} & \multirow{2}{*}{$\mathrm{M} / 4 \mathrm{y}$} & $\mathrm{R}$ & \multirow{2}{*}{ Bilateral hearing loss since childhood } & Profound & - & - \\
\hline & & $\mathrm{L}$ & & Profound & - & - \\
\hline 4 & $F / 6$ y & $\mathrm{R}$ & Hearing loss since childhood & Profound & - & - \\
\hline 5 & $\mathrm{~F} / 6 \mathrm{y}$ & L & Hearing impairment since childhood, persistent tinnitus & Profound & + & - \\
\hline 6 & $\mathrm{~F} / 4 \mathrm{~m}$ & $\mathrm{R}$ & Microtia, EAC atresia & Profound & ND & - \\
\hline 7 & $\mathrm{~F} / 8 \mathrm{y}$ & L & Hearing impairment since childhood & Moderate & - & - \\
\hline 8 & $F / 15$ y & $\mathrm{R}$ & Hearing loss since childhood & Severe & - & - \\
\hline 9 & $\mathrm{~F} / 5 \mathrm{y}$ & $\mathrm{R}$ & Hearing loss since childhood & Profound & - & - \\
\hline 10 & $M / 11$ y & L & Hearing loss since childhood, occasional vertigo & Profound & + & - \\
\hline 11 & $F / 2$ y & L & Microtia, EAC atresia, and suckling and drinking dysfunction since birth & Severe & ND & + \\
\hline 12 & $M / 13$ y & $\mathrm{L}$ & Hearing loss since childhood & Profound & - & - \\
\hline
\end{tabular}

+ means positive, - means negative. $A B R=$ auditory brainstem response, $E A C=$ external auditory canal, $F=$ female, $F N E=$ facial nerve examination, $L=$ left, $M=$ male, $m=$ month, $N D=$ not done, $R=$ right, $y=$ year 


\section{Image Evaluation}

The HRCT (12 DIAC cases) and MRI (9 DIAC cases) were reevaluated independently by 2 experienced otoradiologists. Any disagreement between them was resolved through consensus. The side, bony septum (completeness, thickness [middle], and stereodirection), diameter (middle), connection, and neural components of duplicated canals (VCN,
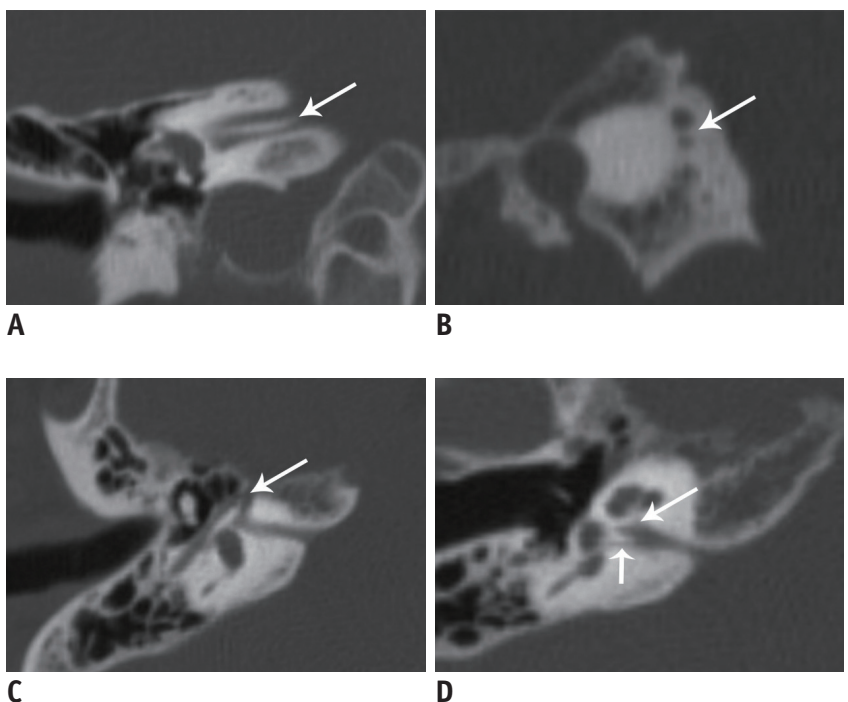

C

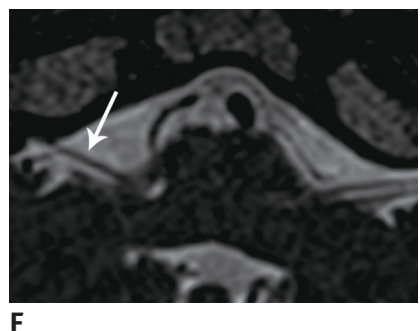

$\mathbf{E}$
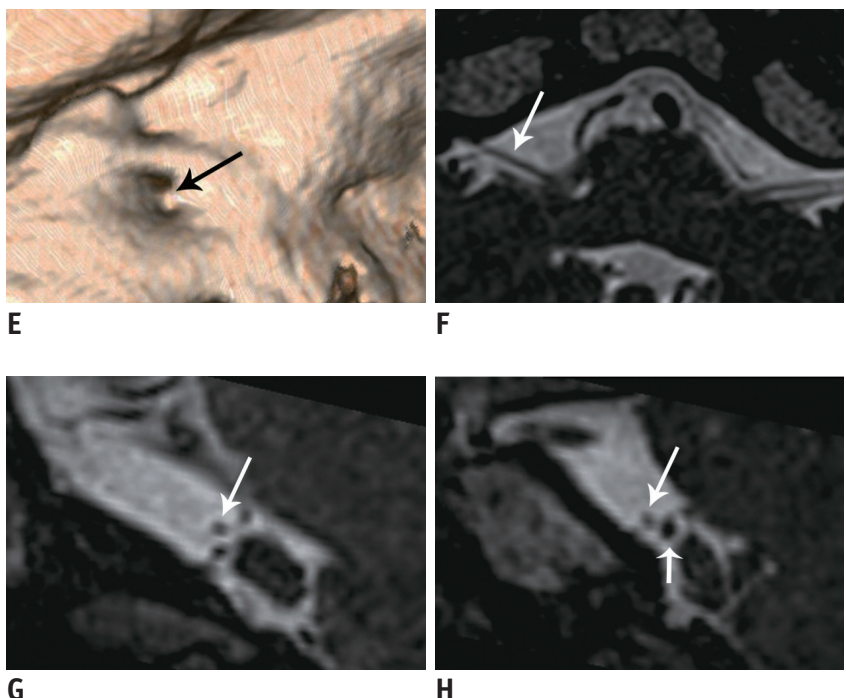

Fig. 1. 23-year-old male with right DIAC.

A, B. Oblique coronal and parasagittal HRCT images show that IAC is divided into double canals by complete horizontal bony septum (arrows). C, D. Superior portion is connected to FN canal (arrows), whereas inferior portion is connected to cochlea and vestibule (short arrow). Ipsilateral bony $\mathrm{CN}$ canal is narrow (long arrow). E. CT volume rendering image shows bony septum (arrow). F-H. Oblique axial and parasagittal MRI show that right VCN (short arrow) is aplastic and FN (long arrows) is normal in cisternal segment. $\mathrm{CN}=$ cochlear nerve, $\mathrm{CT}=$ computed tomography, DIAC = duplicated internal auditory canal, $\mathrm{FN}=$ facial nerve, $\mathrm{HRCT}=$ high-resolution $\mathrm{CT}, \mathrm{IAC}=$ internal auditory canal, $\mathrm{VCN}=$ vestibulocochlear nerve superior/inferior vestibular nerve [SVN/IVN], CN, and FN) and accompanying temporal anomalies (inner, middle, and outer ear anomalies, and FN migration) and systemic anomalies, were recorded. The direction of the bony septum was classified into horizontal, oblique, and vertical directions, based on the lateral semicircular canal. The related diagnostic criteria were as follows: 1) VCN, FN, CN, SVN, and IVN were classified as normal, hypoplastic, aplastic on the oblique axial, coronal, and parasagittal MRI scans (28). 2) On CT images, the bony $\mathrm{CN}$ canal was considered stenotic if its

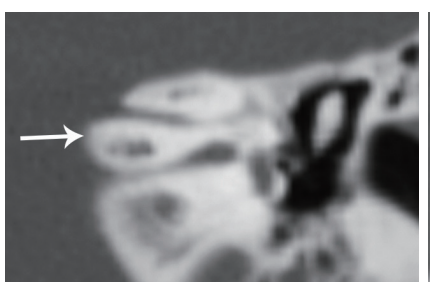

A

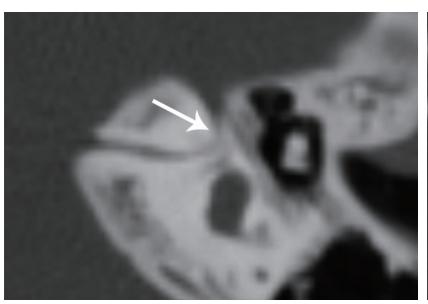

C

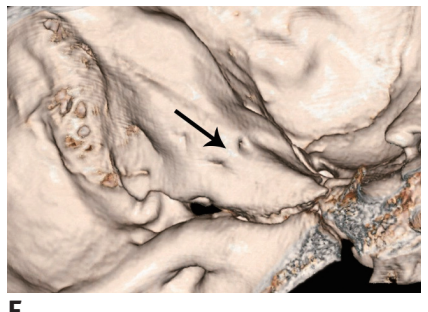

E

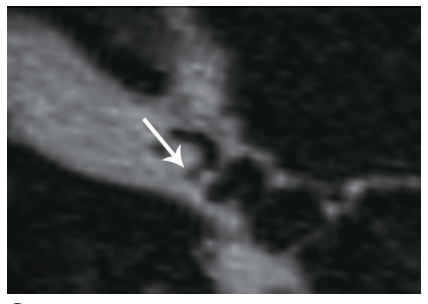

G

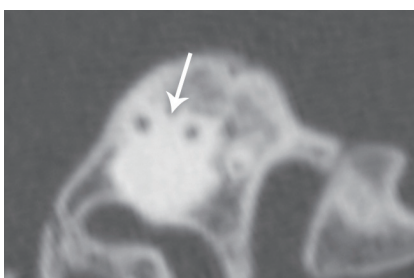

B
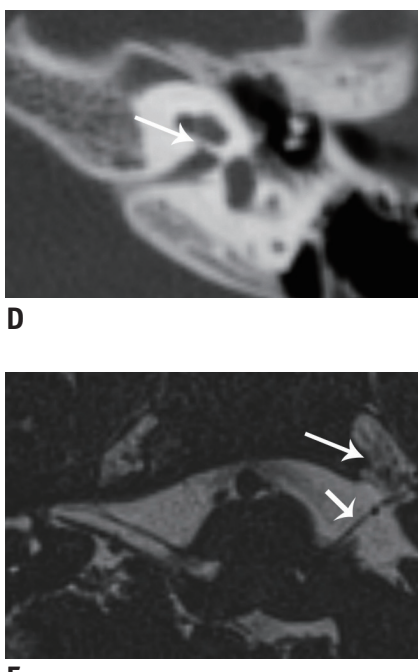

$\mathbf{F}$

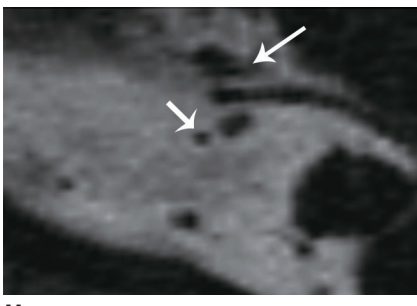

H
Fig. 2. 6-year-old female with left DIAC.

A, B. Oblique coronal and parasagittal HRCT images clearly show complete and nearly vertical bony septum (arrows). C. Anterior canal is connected to FN canal (arrow). D. Posterior canal ends in cochlea and vestibule, and ipsilateral bony CN canal is stenotic (arrow). E. CT volume rendering image clearly shows that FN canal meatus is anteriorly and superiorly located (arrow). F-H. Oblique axial and parasagittal MR images reveal left aplastic VCN and normal FN (short arrows). Latter has migrated beneath trigeminal nerve (long arrows). $\mathrm{MR}=$ magnetic resonance 

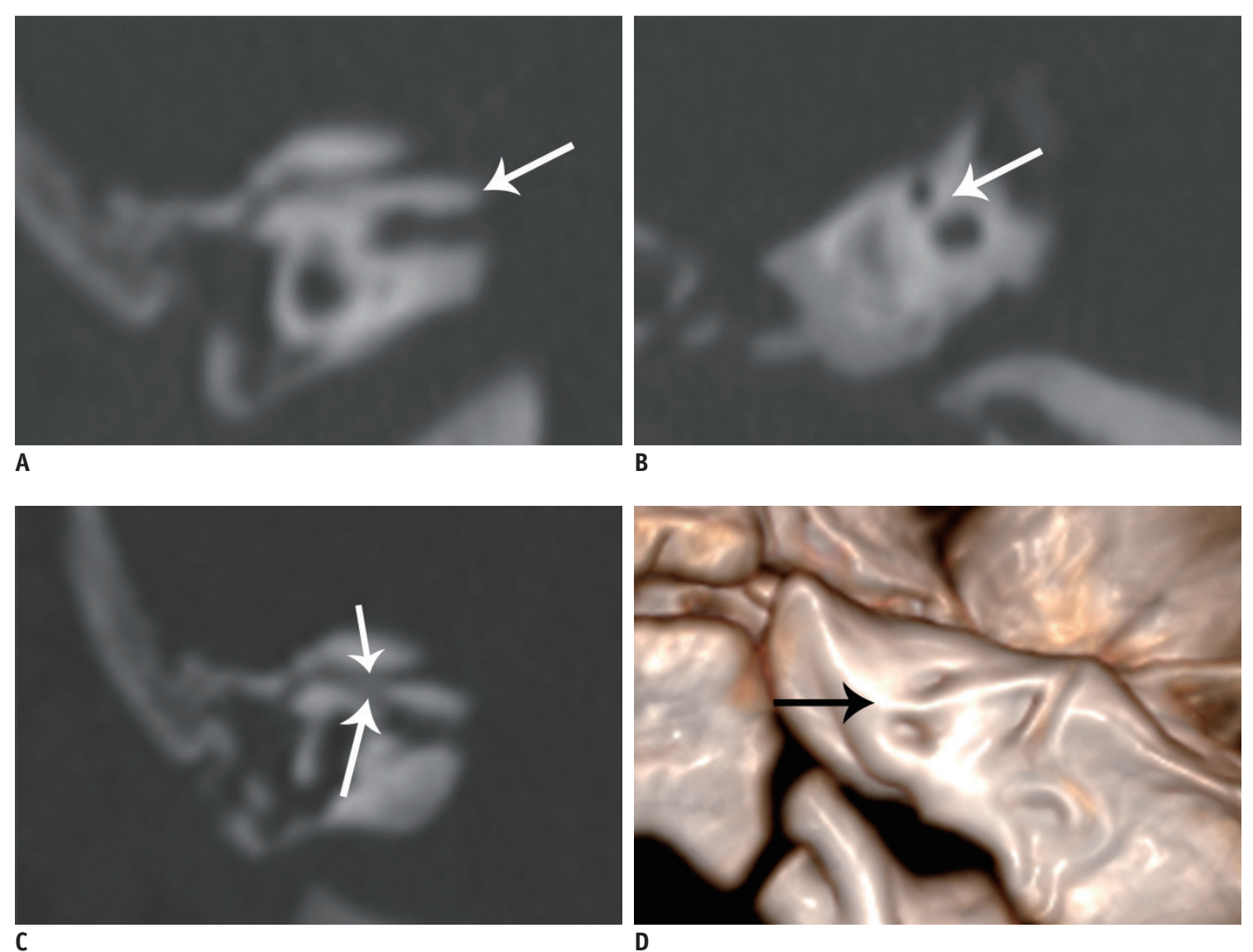

Fig. 3. 4-month-old female with right DIAC.

A, B. Oblique coronal and parasagittal HRCT images show that IAC is divided into two portions by complete oblique bony septum (arrows). C. FN canal is continuous with anterosuperior canal (short arrow) and with posteroinferior canal through accessory canal (long arrow). D. CT volume rendering image shows meatus of double canals and bony septum (arrow).

diameter was $<1.4 \mathrm{~mm}(29)$. 3) The migration of the FN or FN canal was determined according to the method described by Vincenti et al. (16).

\section{RESULTS}

The frequency of DIAC among patients with SNHL was $0.019 \%(12 / 64813)$.

\section{CT Findings}

The 13 explored IACs were divided into double canals by complete $(n=6)$ (Figs. 1-3) and incomplete $(n=7)$ bony septa (Fig. 4). The stereodirection of the bony septum ranged from horizontal (Fig. 1) to approximately vertical (Fig. 2). All of the superoanterior canals were continuous with the labyrinthine segment of the FN canal, except for 1 that also extended to the vestibule (Fig. 4). The diameter ranged from $0.60 \mathrm{~mm}$ to $2.53 \mathrm{~mm}$ (mean: 1.32 $\mathrm{mm})$. The duplicated FN canal meatuses in 2 cases (15.4\%) were anterosuperiorly located (Figs. 2, 3) in the temporal petrous part. The inferoposterior canals were continuous with the cochlea and vestibule, except for 2 that also extended to the FN canals through accessory canals (Fig. 3). The diameter ranged from $0.50 \mathrm{~mm}$ to $2.1 \mathrm{~mm}$ (mean: $1.27 \mathrm{~mm}$ ). All 13 ears were associated with temporal anomalies: inner ear malformations (including a narrow bony $\mathrm{CN}$ canal $[\mathrm{n}=12,92.3 \%$ ] (Figs. 1,2$)$, enlarged vestibules and dysplastic lateral semicircular canals $[\mathrm{n}=$ 3]), and middle and outer ear malformations (each $\mathrm{n}=2$, including ossicle dysplasia, microtia, and EAC atresia). In 1 case, the tympanic cavity was separated from the inner ear by a thick bony wall with oval and round window atresia and anterior migration of the mastoid segment of the FN canal. All of the duplicated canals and bony septa were completely demonstrated on the oblique axial, oblique coronal, and parasagittal CT MPR images. The meatuses of duplicated canals and bony septa (Figs. 1-3) were clearly depicted in 3D volume-rendering images. 


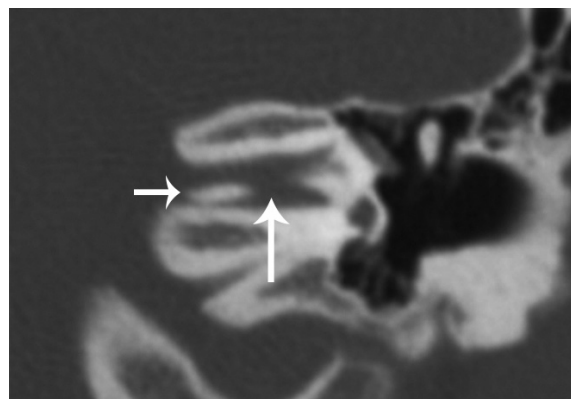

A

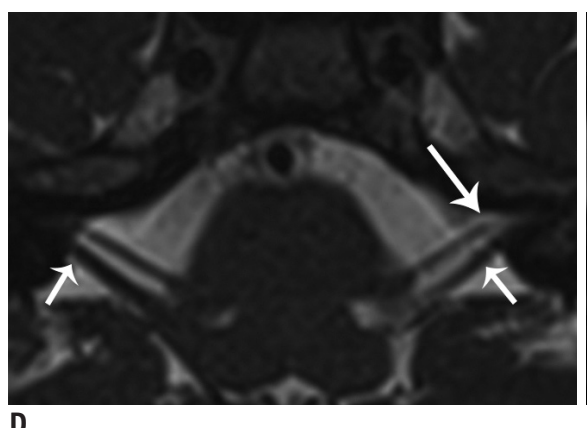

D

Fig. 4. 8-year-old female with left DIAC.

A. Oblique coronal HRCT image shows that bony septum has medial (short arrow) and lateral (long arrow) defects. B. Anterosuperior canal is connected to FN canal (short arrow) and to vestibule (long arrow). C. Posteroinferior canal ends in cochlea (short arrow) and vestibule (long arrow). D-F. Oblique axial and parasagittal MR images reveal that left dysplastic VCN (short arrows) and normal FN enter anterosuperior canal (long arrow).

\section{MRI Findings}

In total, $77.8 \%(7 / 9)$ and $22.2 \%(2 / 9)$ of VCNs were aplastic (Figs. 1, 2) and hypoplastic (Fig. 4), respectively. Furthermore, $88.9 \%$ (8/9) of FNs were normal, except for 1 that was hypoplastic, on MRI. All of the FNs entered the anterosuperior canals, and 2 of them migrated anterosuperiorly beneath the ipsilateral trigeminal nerve (Fig. 2). One of the 2 hypoplastic VCNs, together with its $\mathrm{FN}$, entered the anterosuperior canal (Fig. 4), and the other VCN entered the posteroinferior canal. In the IAC segment, in 2 DIAC patients with hypoplastic VCNs, the hypoplastic SVN and IVN of 1 case and the hypoplastic CN and IVN of the other case, were found in the posteroinferior canals. The remaining posteroinferior canals were vacant. In addition, $33.3 \%(3 / 9)$ of the double canals with enlarged vestibules and the dysplastic lateral semicircular canals could be seen in the volume-rendering images.

\section{DISCUSSION}

The prevalence of DIAC has yet to be reported. In our investigation, the rate of occurrence of DIAC among SNHL patients was $0.019 \%$. Our study demonstrated that relatively narrow double canals, divided by a bony septum in the affected IACs of the DIAC patients, were the characteristic HRCT feature, and the VCNs of the affected ears usually appeared aplastic, with intact FNs, on HRMRI. This observation was consistent with the findings of previous studies $(5,18,24,30)$. Most cases of DIAC were misdiagnosed as IAC stenosis on conventional CT, because of the relatively narrow duplicated canals and incomplete bony septa. Previous studies and our own pilot studies demonstrated that most of the VCNs or CNs in the affected ears of patients with IAC stenosis are dysplastic $(25,31)$, and most of these patients can benefit from ECI; conversely, VCNs or CNs of DIAC patients are aplastic, and most of them are unsuitable for ECI (23). Thus, accurate diagnosis and differentiation between these conditions, preoperatively, are necessary to help otologists choose the appropriate treatment methods.

DIAC affects ears unilaterally, and rarely occurs in bilateral ears (19). It can present as an isolated finding $(7,9)$, occasionally associated with other temporal malformations $(15,18)$ or can form part of some syndromes, such as Klippel-Feil syndrome (11) and pontine tegmental cap dysplasia $(22,23)$. In our patient series, $100 \%$ (13 ears) of DIACs were accompanied by temporal malformations, including $100 \%$ inner ear malformations, $15.4 \%$ middle ear 
anomalies, and $15.4 \%$ outer ear anomalies, but these cases did not have other syndromes. In this series, we found a rare case characterized by separation of the middle ear from the inner ear by a thick bony wall, with oval and round window atresia. No similar case has yet been reported. For DIAC patients requiring ECI, preoperative detection of an accompanying thick bony wall, EAC atresia, FN migration, and round window atresia would be of considerable help to otologists to design appropriate surgical approaches.

In previous studies, patients with DIAC presented a variable degree of hearing dysfunction since childhood; some of them suffered from tinnitus and vertigo $(6,18)$, and they rarely had FN dysfunction (18). In this study, the percentages of hearing, vestibular, and FN dysfunctions were $100 \%(13 / 13), 15.4 \%(2 / 13)$, and $7.7 \%(1 / 13)$, respectively. In this series, profound SNHL was the most common clinical presentation, accounting for $76.9 \%$ (10/13).

Given the complexities of completeness, thickness, and stereodirection of bony septa, and the connection of duplicated canals, it was difficult to reveal them clearly on a single conventional axial, coronal, or sagittal CT image. Our study showed that only three CT MPR images, utilized together, could avoid misdiagnosis and missed diagnosis. In addition, CT volume rendering images could three-dimensionally depict the meatus of duplicated canals and the bony septum. Thus, this modality could be used complementarily with MPR images.

The nerve structures (VCNs and FNs) in the cisternal segment could be easily depicted, while the branches of the VCN and FN, especially dysplastic ones, in the relatively narrow duplicated canals were not easily demonstrated because of insufficient cerebrospinal fluid. In 1 case with a dysplastic VCN, depicted by MRI in this series, the CN could not be shown, although submillimeter HRMRI was applied. Considering their clinical presentation and moderate SNHL, as revealed by an auditory test, we considered that this $\mathrm{CN}$ did exist but might be dysplastic. Thus, the $\mathrm{CN}$ evaluation of patients with DIAC through MRI should be integrated with full auditory test results and clinical presentation to obtain accurate information for the selection of suitable candidates for ECI.

This study had a few limitations. First, the number of DIAC patients was small because of the rarity of this phenomenon. Second, the vestibular function of the affected ears was evaluated with the Caroli test only, which might have been insufficient. As such, more comprehensive tests should be conducted to evaluate vestibular functions in future studies. Finally, although a submillimeter highresolution sequence was applied in our study, some severely hypoplastic VCNs or CNs might be misdiagnosed as aplastic because of the limitation in the spatial resolution of MRI.

In conclusion, DIAC is a rare IAC anomaly that accounts for $0.019 \%$ of SNHL patients. The affected ears usually present profound SNHL. A double-canal appearance is the characteristic feature of DIAC in HRCT scans, and is usually accompanied by other ear anomalies. Conversely, the VCN commonly appears as aplastic, with a normal FN, on HRMRI. Therefore, ECI should be cautiously used in patients with DIAC.

\section{Conflicts of Interest}

The authors have no potential conflicts of interest to disclose.

\author{
ORCID iDs \\ Lihong Zhang \\ https://orcid.org/0000-0002-6347-986X \\ Linsheng Wang \\ https://orcid.org/0000-0002-6893-0322
}

\section{REFERENCES}

1. Clemens F, Sandström J. Double-barreled hypoplastic internal auditory canal in unilateral deafness. Acta Radiol Diagn 1975;16:342-346

2. Goktas BT, Karadag D, Calisir C, Adapinar B. Bilateral narrow duplicated internal auditory canal. Eur Arch Otorhinolaryngol 2008;265:999-1001

3. Curtin H, May M. Double internal auditory canal associated with progressive facial weakness. Am J Otol 1986;7:275-281

4. Weissman JL, Arriaga M, Curtin HD, Hirsch B. Duplication anomaly of the internal auditory canal. AJNR 1991;12:867869

5. Weon YC, Kim JH, Choi SK, Koo JW. Bilateral duplication of the internal auditory canal. Pediatr Radiol 2007;37:1047-1049

6. Ferreira T, Shayestehfar B, Lufkin R. Narrow, duplicated internal auditory canal. Neuroradiology 2003;45:308-310

7. Cho YS, Na DG, Jung JY, Hong SH. Narrow internal auditory canal syndrome: parasagittal reconstruction. J Laryngol Otol 2000;114:392-394

8. Baik HW, Yu H, Kim KS, Kim GH. A narrow internal auditory canal with duplication in a patient with congenital sensorineural hearing loss. Korean J Radiol 2008;9 Suppl:S22-S25

9. Casselman JW, Offeciers FE, Govaerts PJ, Kuhweide R, Geldof $H$, Somers T, et al. Aplasia and hypoplasia of the 
vestibulocochlear nerve: diagnosis with MR imaging. Radiology 1997;202:773-781

10. Vilain J, Pigeolet Y, Casselman JW. Narrow and vacant internal auditory canal. Acta Otorhinolaryngol Belg 1999;53:67-71

11. Demir OI, Cakmakci H, Erdag TK, Men S. Narrow duplicated internal auditory canal: radiological findings and review of the literature. Pediatr Radiol 2005;35:1220-1223

12. Kesser BW, Raghavan P, Mukherjee S, Carfrae M, Essig G, Hashisaki GT. Duplication of the internal auditory canal: radiographic imaging case of the month. Otol Neurotol 2010;31:1352-1353

13. Ramírez-Camacho R, Berrocal JR, Arellano B. Bilateral malformation of the internal auditory canal: atresia and contralateral transverse megacrest. Otolaryngol Head Neck Surg 2001;125:115-116

14. Guirado CR. Malformations of the inner auditory canal. Rev Laryngol Otol Rhinol 1992;13:419-421

15. Lee SY, Cha SH, Jeon MH, Bae IH, Han GS, Kim SJ, et al. Narrow duplicated or triplicated internal auditory canal ( 3 cases and review of literature): can we regard the separated narrow internal auditory canal as the presence of vestibulocochlear nerve fibers? J Comput Assist Tomogr 2009;33:565-570

16. Vincenti V, Ormitti F, Ventura E. Partitioned versus duplicated internal auditory canal: when appropriate terminology matters. Otol Neurotol 2014;35:1140-1144

17. Kono T, Kuwashima S, Arakawa H, Yamazaki E, Kitajima K, Ejima $Y$, et al. Narrow duplicated internal auditory canal: a rare inner ear malformation with sensorineural hearing loss. Arch Otolaryngol Head Neck Surg 2009;135:1048-1051

18. Kew TY, Abdullah A. Duplicate internal auditory canals with facial and vestibulocochlear nerve dysfunction. J Laryngol Otol 2012;126:66-71

19. Kishimoto I, Moroto S, Fujiwara K, Harada H, Kikuchi M, Suehiro A, et al. Bilateral duplication of the internal auditory canal: a case with successful cochlear implantation. Int J Pediatr Otorhinolaryngol 2015;79:1595-1598

20. Binnetoğlu A, Bağlam T, San M, Gündoğdu Y, Batman Ç. A challenge for cochlear implantation: duplicated internal auditory canal. J Int Adv Otol 2016;12:199-201

21. Sakina MS, Goh BS, Abdullah A, Zulfiqar MA, Saim L. Internal auditory canal stenosis in congenital sensorineural hearing loss. Int J Pediatr Otorhinolaryngol 2006;70:2093-2097

22. Desai NK, Young L, Miranda MA, Kutz JW Jr, Roland PS, Booth TN. Pontine tegmental cap dysplasia: the neurotologic perspective. Otolaryngol Head Neck Surg 2011;145:992-998

23. Nixon JN, Dempsey JC, Doherty D, Ishak GE. Temporal bone and cranial nerve findings in pontine tegmental cap dysplasia. Neuroradiology 2016;58:179-187

24. Wang LS, Zhang LH, Sun XH, Yang YY, Chen YQ, Li X, et al. [Imaging features of duplication of the internal auditory canal.] Zhonghua Er Bi Yan Hou Tou Jing Wai Ke Za Zhi 2010;45:481-485

25. Wang LS, Zhang LH, Li XY, Chen YQ, Li X, Sun ZG, et al. [CT and MRI diagnosis of congenital stenosis of the internal auditory canal.] Zhonghua Er Bi Yan Hou Tou Jing Wai Ke Za Zhi 2011;46:533-538

26. Casselman JW, Offeciers EF, De Foer B, Govaerts P, Kuhweide $\mathrm{R}$, Somers T. CT and MR imaging of congenital abnormalities of the inner ear and internal auditory canal. Eur J Radiol 2001;40:94-104

27. Norton SJ, Gorga MP, Widen JE, Folsom RC, Sininger Y, Cone-Wesson B, et al. Identification of neonatal hearing impairment: summary and recommendations. Ear Hear 2000;21:529-535

28. Sennaroglu L, Saatci I. A new classification for cochleovestibular malformations. Laryngoscope 2002;112:2230-2241

29. Stjernholm C, Muren C. Dimensions of the cochlear nerve canal: a radioanatomic investigation. Acta Otolaryngol 2002;122:43-48

30. Giesemann AM, Neuburger J, Lanfermann H, Goetz F. Aberrant course of the intracranial facial nerve in cases of atresia of the internal auditory canal (IAC). Neuroradiology 2011;53:681-687

31. Kariya S, Nishizaki K, Akagi H, Paparella MM. Abnormal direction of internal auditory canal and vestibulocochlear nerve. J Laryngol Otol 2004;118:902-905 